\title{
Reconfigurable Constraint-Based Reactive Framework for Assistive Robotics with Adaptable Levels of Autonomy
}

\author{
Santiago Iregui ${ }^{1}$, Joris De Schutter ${ }^{1}$ and Erwin Aertbeliën ${ }^{1}$.
}

\begin{abstract}
In this work, we propose a constraint-based reactive framework that allows an easy customization of assistive robotic systems to comply with the particular needs of users with different levels of mobility. This framework enables the specification of modular assistive reactive behaviors which can be easily composed to allocate autonomy over robot-related or taskrelated variables. Such assistive behaviors can be systematically modulated online, which results in adaptable levels of autonomy. Modulation can be performed directly by the user through suitable human-machine interfaces, or can be performed automatically by the computer. Although our framework also shows potential for handling other types of tasks, we focus on assisting the user with reaching tasks. We experimentally validated the flexibility and applicability of our framework by presenting three assistive use cases, each targeting users with different level of mobility. Each of those cases was easily reconfigured to include various HMIs (e.g. SpaceMouse, eye tracker, etc), exploiting different assistive strategies. During the experiments, the level of autonomy could be automatically or manually adapted.
\end{abstract}

Index Terms-Physically Assistive Devices, Physical HumanRobot Interaction, Sensor-based Control

\section{INTRODUCTION}

$\mathbf{T}$ HE quality of life of people with upper motor disabilities, often caused by spinal cord injuries, is severely reduced. Assistive robotic solutions offer an opportunity to improve the quality of life of these people. In most cases, a completely autonomous solution is not desired as shown in [1]. From a neuro-rehabilitation point of view, people have a need to keep their remaining autonomy or recover some of their lost autonomy. People also want to feel in control as much as possible. Thus, robotic assistive solutions have been proposed that share autonomy between human and computer, and that can assist persons with specific levels of mobility (henceforth referred to as users) in particular scenarios.

However, these solutions often provide limited possibilities for customization, ignoring individual mobility difficulties and preferences. Often, customization is limited to changing the Human-Machine Interface (HMI) used to drive a subset of the degrees of freedom $(D o F)$ of the robotic manipulator.

Manuscript received: February, 24, 2021; Revised: May, 23, 2021; Accepted: July, 11, 2021

This paper was recommended for publication by Editor Jee-Hwan Ryu upon evaluation of the Associate Editor and Reviewers' comments. All authors gratefully acknowledge the financial support by SmartHandler_ICON and $\mathrm{KU}$ Leuven $\mathrm{C} 1$ project "Neurophysiological investigations of the human and nonhuman primate brain: from perception to action" (3M180301). santiago.iregui@kuleuven.be

${ }^{1}$ The authors are with the Robotics Research Group, KU Leuven and Core Lab ROB, Flanders Make@KU Leuven.

Digital Object Identifier (DOI): see top of this page.
In this work, we introduce a framework that increases the flexibility to customize assistive solutions with robotic manipulators. For this purpose, we encapsulate the allocation of autonomy over decoupled DoFs into a set of building blocks. These building blocks enable continuous reaction to HMI-input and sensor-input measuring changes in the environment, which offers robustness and continuous human-robot interactivity to the application. These building blocks can be (i) composed, which enables the possibility of customizing the assistance according to the particularities of the user and the features of the scenario; and (ii) modulated, such that the level of autonomy over computer-controlled or user-controlled variables can be gradually shifted. The latter can be specified by event HMIs (e.g. buttons) that trigger a transition of certain behaviors, or by a knob-like HMI that allows to manually modulate the desired autonomy.

Autonomy can be allocated not only over decoupled robotrelated DoF, but also over decoupled task-related DoF. This, in combination with the offered composability, is a key advantage of our framework over previous shared autonomy paradigms, as it gives us more customization possibilities. For instance, the user can have autonomy over robot-related DoFs or task-related DoFs through various types of HMIs such as: teleoperation HMIs (such as 2D or 3D joysticks), kinesthetic HMIs that interact with the end effector through direct force interaction, and target HMIs such as eye-trackers that can indicate the end point of a path. All these HMIs can be combined simultaneously in one application.

Thus, the main contribution of this work is an assistive framework that is: (i) adaptable, by enabling a systematic way of transitioning between levels of autonomy over decoupled DoFs; and (ii) reconfigurable, by allowing customization for different users through the combination of different types of HMIs, assistive strategies, robotic platforms, etc. As an additional contribution, we introduce novel assistive behaviors that can be composed within the framework to suit the needs of users with different levels of mobility. We experimentally validated the reconfigurability and adaptability of this framework by demonstrating three use cases, targeted for able-bodied, paraplegic and tetraplegic users, respectively.

Following section II on related work, section III describes the controller used to specify various behaviors, while section IV describes how to modularly create these behaviors through the definition of constraints. The level of autonomy given by the behaviors can be arbitrated by means of the method presented in section $\mathrm{V}$, which decides based on the monitoring 
of parameters such as the user intent estimated in section VI. We present experimental validation in section VII and conclusions in section VIII.

\section{RELATED WORK}

Different assistive solutions have been designed with HMIs suitable for certain limitations of mobility. For instance, in [2], tetraplegic users can use an eye tracker for indicating the desired object through their gaze, which makes the robot automatically follow an offline planned path towards the object. Other approaches, like [3], grant more autonomy to users with more mobility and allow them to control a 7 DoF manipulator in a reduced learned latent space controlled by a $2 \mathrm{D}$ joystick during object reaching tasks. However, the authors conclude that the latent actions became counter-intuitive and erratic when the state of the robot is not near the training data.

The use of virtual guidance fixtures $(V G F)$ has been explored extensively in assistive robotics, while only recently their adaptation to new situations has been explored. For instance, in [4], the authors proposed a tube-shaped VGF that assists users in performing kinesthetic teaching according to previously demonstrated paths. Although the VGF is activated/deactivated when deviating too much from it, its shape cannot be adapted online according to changes in the environment. The same happens with [5], where the authors generate a VGF from straight lines originating from segmented demonstrated paths, which can be automatically switched on/off when deviating from it. A more adaptive approach to comply with changing environments was proposed in [6], where users can online: generate the geometry of a VGF, switch between generated VGFs, and also adapt their pose. Nevertheless, in [6], such adaptations to comply with changing environments are always handled manually by the user. Reinforcement learning approaches have also been explored to online plan and replan (i.e. to adapt) VGFs, such as in [7] where a mixture of ProMPs is used to represent their paths.

Additionally, constraint-based assistive approaches have been proposed for sharing the autonomy between user and computer. Hierarchical constraints were specified in the Stack of Tasks framework in [8], which make the end effector point autonomously towards a target object while the user teleoperates its position. On the other hand, in [9], constraintbased assistive templates were proposed for specifying nonadaptive shared autonomy behaviors, each belonging to a sequential finite state machine that solves a specific task.

Few customization possibilities have been reported in the state-of-the-art. For instance, in the approach of [10], online customization of the mapping function of the HMI is enabled and performed by the user through examples. Similarly, in [11], the user is allowed to customize the level of assistance in a shared autonomy system through verbal cues.

\section{TASK SPECIFICATION AND CONTROL IN ETASL}

In order to specify the behaviors that compose our assistive framework, we use the constraint-based task specification and control framework eTaSL [12]. In $e T a S L^{1}$, the behaviors are expressed by a set of hard (high priority) and soft (low priority) constraints over expressions, which are defined as functions of joint positions $\boldsymbol{q}$, time $t$, and feature variables that are grouped in vector $\chi$. These feature variables are defined as auxiliary free variables that facilitate the specification of DoFs associated with the task. They can be monitored, controlled and/or bounded. eTaSL translates this specification of behavior automatically into the quadratic problem (1):

$$
\begin{array}{ll}
\underset{\dot{\boldsymbol{Q}}, \varepsilon}{\operatorname{minimize}} & \dot{\boldsymbol{Q}}^{\top} \mu \boldsymbol{I} \dot{\boldsymbol{Q}}+\varepsilon^{\top} \boldsymbol{W}_{\varepsilon} \varepsilon \\
\text { subject to } & \boldsymbol{L}_{\boldsymbol{A}} \leq \boldsymbol{A} \dot{\boldsymbol{Q}} \leq \boldsymbol{U}_{\boldsymbol{A}},
\end{array}
$$

where $\boldsymbol{Q}=\left[\begin{array}{ll}\boldsymbol{q}^{\top} & \chi^{\top}\end{array}\right]^{\top}$. For each constraint $i$, a slack variable $\varepsilon_{i}$ (grouped in $\varepsilon=\left[\varepsilon_{1}^{\top}, \ldots \varepsilon_{n}^{\top}\right]^{\top}$ ) is defined, which penalizes deviations from the soft constraint with a weight $w_{i}=\mu+\hat{w}_{i}$ (grouped in the diagonal matrix $\boldsymbol{W}_{\varepsilon}$ ). For hard constraints $\varepsilon_{i}=\mathbf{0}$. The regularization factor $\mu$ punishes large $\|\dot{\boldsymbol{Q}}\|^{2}$ in case of an underdetermined set of constraints or in the neighborhood of singularities. The constraints (1b) ensure that task expressions $\boldsymbol{e}_{i}(\boldsymbol{q}, \boldsymbol{\chi}, t)$ evolve to a setpoint $\boldsymbol{e}_{s_{i}}$ by following the first order system (2) with time constant $k_{i}^{-1}$ (denoted as $\boldsymbol{e}_{i} \rightarrow \boldsymbol{e}_{s_{i}}$ ) or evolve to a desired velocity $\boldsymbol{v}_{i}$ (3) (denoted as $\dot{\boldsymbol{e}}_{i} \rightarrow \boldsymbol{v}_{i}$ ).

$$
\begin{aligned}
& \boldsymbol{J}_{i} \dot{\boldsymbol{Q}}=-k_{i}\left(\boldsymbol{e}_{i}-\boldsymbol{e}_{s_{i}}\right)-\partial \boldsymbol{e}_{i} / \partial t+\boldsymbol{\varepsilon}_{i}, \\
& \boldsymbol{J}_{i} \dot{\boldsymbol{Q}}=\boldsymbol{v}_{i}-\partial \boldsymbol{e}_{i} / \partial t+\boldsymbol{\varepsilon}_{i},
\end{aligned}
$$

where $\boldsymbol{J}_{i}=\partial \boldsymbol{e}_{i} / \partial \boldsymbol{Q}$ determine the rows of $\boldsymbol{A}$. For inequality constraints, the equal sign in (2) and (3) is replaced with $\geq$ or $\leq$. The right hand side terms of (2) and (3) determine the limits $\boldsymbol{L}_{\boldsymbol{A}}$ and/or $\boldsymbol{U}_{\boldsymbol{A}}$. eTaSL performs automatic differentiation to calculate all the involved derivatives, facilitating the reconfigurability of the system. See [12] for details about the derivation of (2) and (3) and the numerical solution of (1).

\section{ReACtive Assistive BehaViors}

This section explains how different assistive strategies can be specified using two types of building blocks (BB) which, when combined, create reactive behaviors. The first type, referred to as reactive action model (RAM), consists of a set of constraints and variables (task-related and/or robot-related) that define a nominal action (e.g. follow a path) and the ability to deviate from it according to these variables. The second type, referred to as sensor interaction model (SIM), consists of a set of constraints that define the interaction between the variables in the RAMs and a sensor or HMI. Both desired and unintended effects that the BBs produce onto each other (e.g. due to environmental disturbances measured with the sensors) are referred to as interactions (see Fig. 1). This separation into the two types facilitates the reconfiguration of BBs to assist a user with particular limitations in a task, using suitable HMIs and sensors.

The connection of these two types of $\mathrm{BBs}$ with the rest of the framework is shown in Fig. 1. The constraints defining the BBs explained in this section are listed in Table I, which are then assembled by eTaSL to automatically generate the controller. To keep the explanation concise, the dependencies of the expressions $e_{i}$ are only explicitly mentioned when needed for clarity.

${ }^{1}$ Open source code, tutorials, examples and a template compatible with multiple commercial robots available at https://etasl.pages.gitlab.kuleuven.be/. 


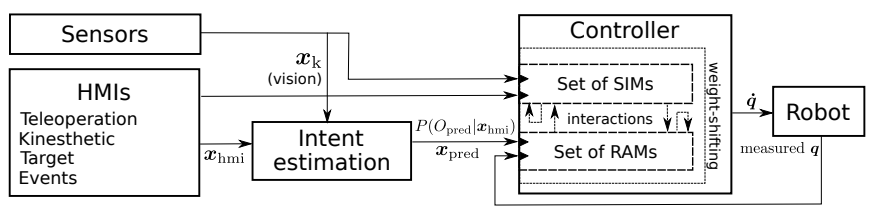

Fig. 1. A scheme of how the components of the framework are connected. The symbols denoting the signals are explained in section VI.

\section{A. Sensor interaction models (SIMs)}

SIMs not only enable the direct interaction between the sensed data and the robot itself, but also with adaptive paths whose shapes can change online. This section presents a nonexclusive list of SIMs, useful for different applications.

Position SIM: Constraints in the form of $\boldsymbol{e}_{i} \rightarrow \tilde{\boldsymbol{R}}_{\mathrm{p}} \boldsymbol{x}_{\mathrm{s}}$ can enforce any expression $e_{i}$ in $\mathbb{R}^{N_{p}}$ to follow position sensor data $\boldsymbol{x}_{\mathrm{s}}$, mapped by a transformation expression $\tilde{\boldsymbol{R}}_{\mathrm{p}}$. For instance, constraint 4 allows the user to control the end position $\left.\boldsymbol{f}_{p}\right|_{s=1}$ of the adaptive path $f_{\mathrm{p}}$ (defined in subsection IV-B) through the target HMI whose position input is $\boldsymbol{x}_{\mathrm{hmi}}$. In addition, collision avoidance is implemented by bounding (in)directly measured distances to obstacles using inequality constraints. For instance, constraint 5 constitutes a path obstacle avoidance SIM by keeping distances $d_{i}$, measured from an obstacle to $j$ protective spheres located along $f_{\mathrm{p}}$ with equidistant values of the arc length, above a threshold $d_{\text {th }}$. Similarly, a tool obstacle avoidance SIM (constraint 6 ) keeps distances $\tilde{d}_{i}$, from an obstacle to $h$ protective spheres distributed along the robot tool, above a threshold $\tilde{d}_{\mathrm{th}}$.

Velocity SIM: Constraints in the form of $\dot{\boldsymbol{e}}_{i} \rightarrow \tilde{\boldsymbol{R}}_{v} \boldsymbol{v}_{\mathrm{s}}$ enforce the velocity of any expression $\boldsymbol{e}_{i}$ in $\mathbb{R}^{N_{v}}$ to follow velocity sensor data $\boldsymbol{v}_{\mathrm{s}} \in \mathbb{R}^{N_{v}}$, mapped by a transformation expression $\tilde{\boldsymbol{R}}_{v}$. For instance, constraint 7 enables the end effector, whose position is denoted by $\boldsymbol{p}(\boldsymbol{q})$, to be controlled by a teleoperation HMI such as a joystick. This can be performed in the world frame $\left(\tilde{\boldsymbol{R}}_{v}=\boldsymbol{I}\right)$ or in the tool frame.

Force SIM: Admittance control reacting to measured forces $\boldsymbol{F}_{\text {meas }}$ in a frame with orientation $\tilde{\boldsymbol{R}}_{\mathrm{f}}$, can be specified using constraints of the form of $\dot{\boldsymbol{e}}_{i} \rightarrow \widetilde{\boldsymbol{R}}_{\mathrm{f}} k_{\text {gain }} \boldsymbol{C}_{a} \boldsymbol{F}_{\text {meas }}$, with a compliance matrix $\boldsymbol{C}_{a}$ and a control gain $k_{\text {gain }}$ [13]. For instance, constraint 8 performs an admittance control in the tool frame, which has orientation $\boldsymbol{R}(\boldsymbol{q})$.

\section{B. Adaptive paths (RAMs)}

An adaptive path that changes its shape through the specification of two RAMs is generated using the method of [14]. The first RAM allows the adaptation of a nominal path to reach changing target positions. This path preserves the encoded shapes that are obtained through a Learning from Demonstration (LfD) method based on Probabilistic Principal Component Analysis (PPCA). This LfD method pre-processes offline a small number of demonstrations (e.g. five) by reparameterizing them with respect to a progress variable $s$, and then generates $M$ basis functions represented in the columns of $\boldsymbol{W}(s)$ [13]. These basis functions encode the learned information and are used during execution to generate an adaptive path $\boldsymbol{f}_{\mathrm{lm}}(\mathrm{lm}=$ learned model $)$ in $\mathbb{R}^{3}$ :

$$
\boldsymbol{f}_{\mathrm{lm}}\left(s, \boldsymbol{\chi}_{\mathrm{lv}}\right)=\boldsymbol{W}(s) \boldsymbol{\chi}_{\mathrm{lv}}+\boldsymbol{b}(s),
$$

TABLE I. Specification of BBs that create the reactive behaviors used in the validation use-cases. Hard constraints are indicated by the omission of $\hat{w}_{i}$.

\begin{tabular}{|c|c|c|c|}
\hline \# & Expression $e_{i}$ & Params & Constraints \\
\hline 1) & $\begin{array}{c}\chi_{\mathrm{lv}} \\
s\end{array}$ & $\begin{array}{c}\hat{w}_{1} \\
k_{2}>0\end{array}$ & $\begin{array}{l}\text { RAM, reactive nominal path }(4) \\
\dot{\boldsymbol{e}}_{1} \rightarrow \mathbf{0} \text { : only adapt shape when needed. } \\
0 \leq e_{2} \leq 1 \text { : limits on the progress. }\end{array}$ \\
\hline 3) & {$\left[\chi_{N}^{\top}, \chi_{B}^{\top}\right]^{\top}$} & $\begin{array}{c}\hat{w}_{3} \\
k_{3}>0\end{array}$ & $\begin{array}{l}\boldsymbol{e}_{3} \rightarrow \mathbf{0}: \text { RAM, nominal path is } \\
\text { preferred in }(5) \text {. }\end{array}$ \\
\hline 4) & $\left.\boldsymbol{f}_{p}\right|_{s=1}-\boldsymbol{x}_{\mathrm{hmi}}$ & $\begin{array}{c}\hat{w}_{4} \\
k_{4}>0\end{array}$ & $\boldsymbol{e}_{4} \rightarrow \mathbf{0}$ : SIM, user controlling $\left.\boldsymbol{f}_{p}\right|_{s=1}$. \\
\hline 5) & {$\left[d_{1}, d_{2}, \ldots d_{j}\right]^{\top}$} & $\begin{array}{c}\hat{w}_{5} \\
k_{5}>0\end{array}$ & $\begin{array}{l}e_{5 i} \geq d_{\text {th }}, \forall i=1, \ldots j: \text { SIM, path } \\
\text { avoiding obstacles. }\end{array}$ \\
\hline 6) & {$\left[\tilde{d}_{1}, \tilde{d}_{2}, \ldots \tilde{d}_{h}\right]^{\top}$} & $\begin{array}{c}\hat{w}_{6} \\
k_{6}>0\end{array}$ & $\begin{array}{l}e_{6 i} \geq \tilde{d}_{\mathrm{th}}, \forall i=1, \ldots h: \text { SIM }, \text { tool } \\
\text { avoiding obstacles. }\end{array}$ \\
\hline 7) & $p(q)$ & $\hat{w}_{7}$ & $\dot{e}_{7} \rightarrow \boldsymbol{v}_{\mathrm{hmi}}:$ SIM, end effector velocity. \\
\hline 8) & $p(q)$ & $\hat{w}_{8}$ & $\begin{array}{l}\dot{\boldsymbol{e}}_{8} \rightarrow \boldsymbol{R}(\boldsymbol{q}) k_{\mathrm{gain}} \boldsymbol{C}_{a} \boldsymbol{F}_{\text {meas }}: \text { SIM, end } \\
\text { effector force. }\end{array}$ \\
\hline 9) & $\left.\boldsymbol{f}_{p}\right|_{s=1}-\boldsymbol{x}_{\text {pred }}$ & $\begin{array}{c}\hat{w}_{9} \\
k_{9}>0\end{array}$ & $\begin{array}{l}e_{9} \rightarrow \mathbf{0}: \text { RAM, attraction to } \\
\text { intent-inferred targets. }\end{array}$ \\
\hline 10) & $\hat{\boldsymbol{n}}_{R}$ & $\begin{array}{c}\hat{w}_{10} \\
k_{10}>0\end{array}$ & $\begin{array}{l}e_{10} \rightarrow 0: \text { RAM, automatic orientation } \\
\text { path. }\end{array}$ \\
\hline $\begin{array}{l}\text { 11) } \\
\text { 12) }\end{array}$ & $\left\|\boldsymbol{p}(\boldsymbol{q})-\boldsymbol{f}_{p}\right\|$ & $\begin{array}{c}\hat{w}_{11} \\
k_{11}>0 \\
\hat{w}_{12}\end{array}$ & $\begin{array}{l}\text { RAM, RVGF } \\
e_{11} \leq r_{\text {tube }}(s) \text { : remain in tube. } \\
\dot{e}_{12} \rightarrow 0 \text { : dampen } s \text {. }\end{array}$ \\
\hline 14) & $\boldsymbol{p}(\boldsymbol{q})-\boldsymbol{f}_{p}$ & $\begin{array}{c}\hat{w}_{13} \\
\hat{w}_{14} \\
k_{14}>0\end{array}$ & $\begin{array}{l}\text { RAM, Auto. reactive approach motion } \\
\dot{e}_{13} \rightarrow V_{p}(s): \text { follow desired velocity } \\
\text { along the path. } \\
\boldsymbol{e}_{14} \rightarrow \mathbf{0} \text { : robot follows path. }\end{array}$ \\
\hline $\begin{array}{l}15) \\
16)\end{array}$ & $\begin{array}{l}q \\
q\end{array}$ & $\begin{array}{l}k_{15}>0 \\
k_{16}>0\end{array}$ & $\begin{array}{l}\text { RAM, joint limits } \\
\boldsymbol{q}_{\min } \leq \boldsymbol{e}_{15} \leq \boldsymbol{q}_{\max } \text { : position limits. } \\
\dot{\boldsymbol{q}}_{\min } \leq \dot{\boldsymbol{e}}_{16} \leq \dot{\boldsymbol{q}}_{\max } \text { : velocity limits. }\end{array}$ \\
\hline
\end{tabular}

where $\boldsymbol{b}(s)$ corresponds to the mean path of the demonstrations, and $\chi_{\mathrm{lv}} \in \mathbb{R}^{M}$ contains feature variables (i.e. which are included in $\chi$ ) representing latent variables of the learned model whose time derivative is constrained with constraint 1 in Table I. The normalized progress variable $s$, whose value is zero at the beginning and one at the end of the path (bounded with constraint 2), is included as a feature variable.

The second RAM defines a path $f_{\mathrm{p}}$ in $\mathbb{R}^{3}$ that can locally deviate from $f_{\mathrm{lm}}$ and adapt to different disturbances specified through SIMs. This adaptability is achieved by including $\chi_{\text {path }}=\left[\begin{array}{lll}\chi_{\mathrm{lv}}^{T} & \chi_{N}^{T} & \chi_{B}^{T}\end{array}\right]^{T}$ as feature variables. Thus,

$$
\boldsymbol{f}_{\mathrm{p}}\left(s, \boldsymbol{\chi}_{\mathrm{path}}\right)=\boldsymbol{f}_{\mathrm{lm}}+\sum_{h=1}^{H} B_{s_{h}}\left(\chi_{N_{h}} \hat{\boldsymbol{N}}_{f}+\chi_{B_{h}} \hat{\boldsymbol{B}}_{f}\right),
$$

where the bell-shaped functions $B_{s_{h}}(s)$ are centered at $H$ instances $s=s_{h}$ along $\boldsymbol{f}_{\operatorname{lm}}\left(s, \boldsymbol{\chi}_{\mathrm{lv}}\right)$. Their amplitudes are modulated by $\chi_{N}=\left[\chi_{N_{1}}, \ldots \chi_{N_{H}}\right]^{\top}$ and $\chi_{B}=\left[\chi_{B_{1}}, \ldots \chi_{B_{H}}\right]^{\top}$, respectively in directions $\hat{\boldsymbol{N}}_{f}\left(s_{h}\right)$ and $\hat{\boldsymbol{B}}_{f}\left(s_{h}\right)$ perpendicular to the tangent of the nominal path at $s_{h}$. Constraint 3 gives these feature variables a preferred value, such that it deforms elastically due to disturbances (similar to [15]).

Not only human demonstrations, as in [13] and [14], but also artificial demonstrations (e.g. from modeled or planned paths) can be used, depending on the application at hand. 


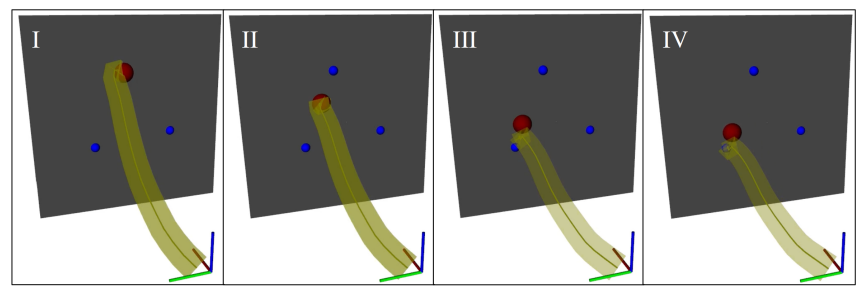

Fig. 2. Snapshots of how the RVGF (translucent yellow) adapts its shape when continuously shifting $\boldsymbol{x}_{\mathrm{hmi}}$ (red sphere). The blue spheres represent known objects and the opaque yellow path depicts $f_{\mathrm{p}}$.

\section{Attraction to intent-inferred targets (RAM)}

The end position $\left.\boldsymbol{f}_{p}\right|_{s=1}$ of path $\boldsymbol{f}_{\mathrm{p}}\left(s, \boldsymbol{\chi}_{\text {path }}\right)$ can be constrained to an intent-inferred position $\boldsymbol{x}_{\text {pred }}$ by means of constraint 9. This RAM grants the computer autonomy over this end position, while the SIM corresponding to constraint 4 gives autonomy to the user. With both BBs active, the autonomy is shared and determined by the weight ratio $\hat{w}_{4} /\left(\hat{w}_{4}+\hat{w}_{9}\right)$. By modulating these weights in function of the posterior probability $P\left(O_{\text {pred }} \mid x_{\mathrm{hmi}}\right)$ of the user wanting to grasp the predicted object $O_{\text {pred }}$ given an input $\boldsymbol{x}_{\mathrm{hmi}}$, we obtain a behavior that assists the user by attracting the end position of the path towards predicted objects (see Fig. 2). If we do not consider effects caused by other BBs, the end position will be determined by the weighted average $\left.\boldsymbol{f}_{p}\right|_{s=1}=\left(\hat{w}_{4} \boldsymbol{x}_{\text {hin }}+\hat{w}_{9} \boldsymbol{x}_{\text {pred }}\right) /\left(\hat{w}_{4}+\hat{w}_{9}\right)$. The modulation of the weights is explained in section $\mathrm{V}$ and the intent-estimation algorithm in section VI.

\section{Automatic orientation path (RAM)}

The orientation of the end effector $\boldsymbol{R}(\boldsymbol{q})$ can be constrained to follow a custom orientation path $\hat{\boldsymbol{R}}(s)$ by means of constraint 10. As an example, the following specifies an axisangle interpolation between an initial orientation $\boldsymbol{R}_{0}$ and a final orientation $\boldsymbol{R}_{1}$. The axis-angle representation $(\hat{\boldsymbol{n}}, \hat{\theta})$ is determined by $[\hat{\boldsymbol{n}} \times] \hat{\theta}=\log \left(\boldsymbol{R}_{0}^{-1} \boldsymbol{R}_{1}\right)$, with $[\hat{\boldsymbol{n}} \times]$ a skewsymmetric matrix corresponding to a normalized vector $\hat{\boldsymbol{n}}$, and $\hat{\theta}$ a scalar angle. The orientation path can be computed as $\hat{\boldsymbol{R}}(s)=\boldsymbol{R}_{0} \exp \left([\hat{\boldsymbol{n}} \times] f_{\hat{\theta}}(s)\right)$ with $0 \leq s \leq 1$. A smooth evolution of the angle $f_{\hat{\theta}}$ that ensures zero derivatives at $s=0$ and $s=1$ can be given, for example, by the cubic polynomial $f_{\hat{\theta}}(s)=3 \hat{\theta} s^{2}-2 \hat{\theta} s^{3}$.

We can apply this orientation path $\hat{\boldsymbol{R}}(s)$ as a constraint on the robot orientation $\boldsymbol{R}(\boldsymbol{q})$ using the task function $\hat{\boldsymbol{n}}_{R} \rightarrow 0$ :

$$
\left[\hat{\boldsymbol{n}}_{R} \times\right]=\log \left(\boldsymbol{R}(q)^{-1} \hat{\boldsymbol{R}}(s)\right) .
$$

Notice that if the end effector advances or recedes through the path $\boldsymbol{f}_{\mathrm{p}}(s)$, the progress variable $s$ gets updated and thus the orientation changes automatically.

\section{E. Reactive Virtual Guidance Fixtures (RAM)}

We propose the generation of a tube-shaped Reactive Virtual Guidance Fixture (RVGF) that adapts its shape due to disturbances and guides the user to the target. In this RAM, a tube with radius $r_{\text {tube }}(s)$ wraps around the nominal path $\boldsymbol{f}_{\mathrm{p}}$ and attracts the position of the end effector $\boldsymbol{p}(\boldsymbol{q})$ towards its interior, where the user can freely move the end effector. This RAM consists of an inequality constraint $\left\|\boldsymbol{p}(\boldsymbol{q})-\boldsymbol{f}_{p}\right\| \leq r_{\text {tube }}(s)$ (see constraint 11), and results in a non-linear impedance-like

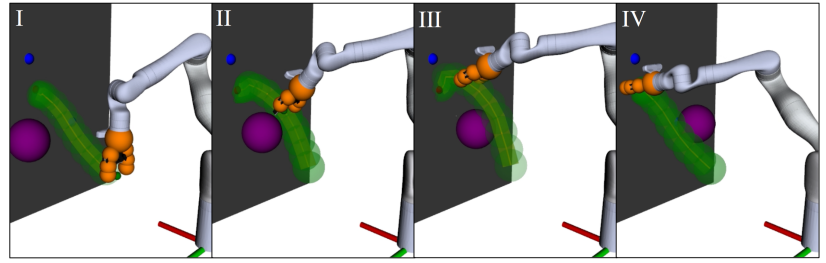

Fig. 3. Snapshots the RVGF adapting to a dynamic obstacle (purple sphere) in human-in-the-loop simulation. Protective spheres in the tool and in the RVGF are shown orange and green, respectively.

guidance when combined with e.g. a velocity or a force SIM. At the same time, this constraint ensures that the value of $s$ is adapted whenever $\boldsymbol{p}(\boldsymbol{q})$ changes due to a disturbance. An additional constraint 12 dampens the motion of $s$ along the path. Notice that the value of $s$ will only be updated when soft constraint 11 is violated, and thus a backlash effect that depends on the magnitude of $r_{\text {tube }}$ exists. When $r_{\text {tube }}$ is large enough, this can cause an undesired lag on other task expressions that depend on $s$, such as $\hat{\boldsymbol{R}}(s)^{2}$.

Fig. 3 shows how this RAM, in combination with the previously defined BBs, guides a user through the RVGF, whose shape reactively adapts to avoid a dynamic obstacle. For this example, $r_{\text {tube }}(s)$ was defined as a piecewise function that is constant throughout $0 \leq s \leq s_{a}$, then linearly decreases to zero at $s_{a} \leq s \leq s_{b}$, and keeps a zero-value near the target position throughout $s_{b} \leq s \leq 1$. In addition, Fig. 2 shows how the RVGF is attracted to the intent-inferred targets due to the behavior described in subsection IV-C.

\section{F. Automatic Reactive Approach Motion (RAM)}

At an arbitrary time, the user may want to grant more autonomy to the computer. For this purpose, two constraints enable the robot to move autonomously from an arbitrary starting position $\boldsymbol{p}_{0}$ to the target while reacting to disturbances caused by other BBs. Constraint 13 drives $s$ from an arbitrary starting value $s_{0}$ towards 1 by imposing a velocity profile $V_{p}(s)$. Constraint 14 makes the end effector slowly approach $f_{\mathrm{p}}(s)$ (due to a low $k_{14}$ ).

Fig. 4 shows a diagram that depicts the obtained behavior. While not using it for specification or execution, for analysis purposes, we present a simplified dynamic model that includes this RAM, plus constraints 7 and 12 that allow us to drive the dynamics to zero (with $\boldsymbol{v}_{\mathrm{hmi}}=0$ since the user is excluded from this analysis). We assume that the Jacobian of end effector does not loose row rank and we omit other constraints such as joint limits, so that the state of the simplified system is purely determined by feature variables $\boldsymbol{Q}=\boldsymbol{\chi}=\left[\begin{array}{ll}\boldsymbol{p}^{\top} & s\end{array}\right]^{\top}$. By substituting the slack variables of the objective function $O_{b}$ in (1) using (2) and (3), and explicitly solving $\partial O_{b} / \partial \dot{\boldsymbol{Q}}=\mathbf{0}$, we obtain:

$$
\begin{aligned}
& \dot{\boldsymbol{p}}=\frac{w_{14}}{\mu+w_{7}+w_{14}}\left(\frac{\partial \boldsymbol{f}_{p}}{\partial s} \dot{s}-k_{14}\left(\boldsymbol{p}-\boldsymbol{f}_{p}(s)\right)\right), \\
& \dot{s}=\frac{w_{14} \frac{\partial \boldsymbol{f}_{p}{ }^{\top}}{\partial s}\left(\dot{\boldsymbol{p}}+k_{14}\left(\boldsymbol{p}-\boldsymbol{f}_{p}(s)\right)\right)+w_{13} V_{p}(s)}{\left(\mu+w_{14} \frac{\partial \boldsymbol{f}_{p}}{\partial s} \frac{\partial \boldsymbol{f}_{p}}{\partial s}+w_{12}+w_{13}\right)} .
\end{aligned}
$$

${ }^{2}$ In these cases, the following equality constraint can be added to eliminate the backlash: $\left(\boldsymbol{p}(\boldsymbol{q})-\boldsymbol{f}_{p}(s)\right) \cdot \boldsymbol{x}_{\tan }(s)=0$, where the tangential vector of the path can be found with $\boldsymbol{x}_{\mathrm{tan}}=\frac{d f_{\mathrm{p}}}{d s} /\left\|\frac{d f_{\mathrm{p}}}{d s}\right\|$. 


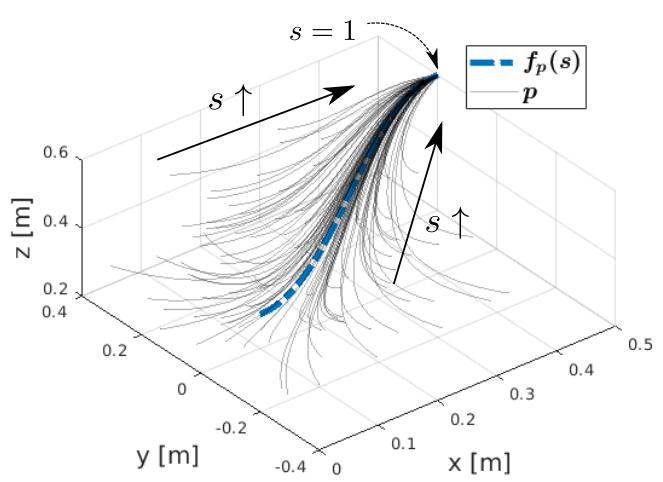

Fig. 4. Multiple paths obtained by solving the simplified dynamics (7) with several initial values of $\boldsymbol{p}$, with $k_{14}=0.7 s^{-1}$ and a fixed path $\boldsymbol{f}_{p}(s)$, without the presence of disturbances over $s$ and $\boldsymbol{p}$.

Equation (7a) shows that $\dot{p}$ will reduce the error of $\boldsymbol{e}_{14}$ proportionally to the corresponding conflicting weights, and such behavior can be turned off by simply setting $w_{14}=0$. The term $\dot{s} \partial f_{p} / \partial s=\dot{\boldsymbol{f}}_{p}$ in (7a) is a feedforward term that substantially improves the tracking error of $e_{14}$.

On the other hand, (7b) shows that if $\hat{w}_{14}=0$, then $\dot{s} \approx w_{13} /\left(\mu+w_{12}+w_{13}\right) V_{p}(s)$. This means that $\dot{s}$ will also track the velocity profile $V_{p}$ proportionally to the corresponding conflicting weights. Nevertheless, when constraint 14 is on, $\dot{s}$ will adapt to disturbances over $\boldsymbol{p}$ based on $\partial \boldsymbol{f}_{p} / \partial s \cdot\left(\boldsymbol{p}-\boldsymbol{f}_{p}\right)$, which can be seen as the tracking error of $\boldsymbol{p}$ projected in the tangent direction of the path, and also based on the corresponding feedforward term $\partial \boldsymbol{f}_{p} / \partial s \cdot \dot{\boldsymbol{p}}$.

Fig. 4 shows an example of several numerical solutions of $\boldsymbol{p}$, obtained by solving (7) with different initial positions $\boldsymbol{p}_{0}$ and a fixed path. Since constraints 2 and 11 where omitted, $s_{0}$ was estimated offline by minimizing $\left\|\boldsymbol{p}_{0}-\boldsymbol{f}_{p}(s)\right\|^{2}$ subject to $0 \leq s \leq 1$ (notice the equivalence). The velocity profile $V_{p}(s)$ was defined as a quasi-trapezoidal profile, whose details will be given in the next section.

\section{LEVELS OF AUTONOMY}

In this work, arbitration is performed by modulating the weights of the constraints that constitute the different BBs. The concept of arbitration is defined in [16]. In this section we define a systematic and modular approach for modulation, based on the concept of level of autonomy.

A level of autonomy is an aspect of the task that can be arbitrated between the user or the robot, e.g. the choice between the user moving freely within an RVGF versus an autonomous motion. An assistive application can have multiple levels of autonomy, each arbitrating the autonomy over some aspects specified by certain BBs. Each level of autonomy can influence the weight of multiple BBs and multiple levels of autonomy can influence the same BB.

Each level of autonomy has an arbitration parameter $G_{k}$ that indicates whether the user and/or the robot will have autonomy, such as the distance to the center of the RVGF or the magnitude of an applied force. These parameters can also be directly controlled by the user discretely (e.g. with an event HMI) or continuously (e.g. with a knob).

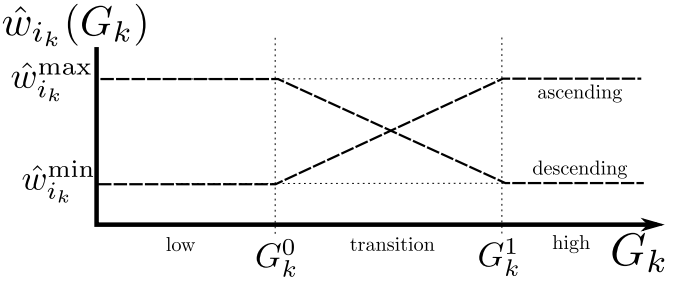

Fig. 5. Definition of the ascending or descending piecewise-continuous function $\hat{w}_{i_{k}}\left(G_{k}\right)$ in terms of the parameters $G_{k}^{0}, G_{k}^{1}, \hat{w}_{i_{k}}^{\min }$, and $\hat{w}_{i_{k}}^{\max }$

This arbitration architecture is modeled by splitting up each of the weights $\hat{w}_{i}$ in Table I into a number of weight factors $\hat{w}_{i_{k}}$ that are function of an arbitration parameter $G_{k}$ :

$$
\hat{w}_{i}=b_{i}+a_{i} \prod_{k \in \Gamma_{i}} \hat{w}_{i_{k}}\left(G_{k}\right),
$$

where $a_{i}$ can be used to scale the product and $b_{i}$ can be used to avoid driving $\hat{w}_{i}$ completely to zero. $\Gamma_{i}$ is the set of subscripts of weight factors that influence the weight $\hat{w}_{i}$. In [14] some intuitive heuristics are specified for selecting the maximum and minimum values of $\hat{w}_{i}$. The weight factors are a piecewise-continuous function of $G_{k}$ as defined in Fig. 5 and can be ascending or descending.

Two policies can be used to determine the values of $G_{k}$ at each time instant: i) time-policy, where $G_{k}$ starts to increase or decrease linearly in time from a fixed value whenever a triggering event occurs (e.g. through an event HMI); and ii) generic-policy, where $G_{k}$ takes the value of any taskrelated or user-related parameter or expression occurring in the specification of the BBs.

Table II presents an example of five different levels of autonomy, each arbitrating the autonomy between user and computer over different aspects of a reaching application. These levels of autonomy are explained as follows:

1. $G_{1}-$ Manual (desc.) versus automatic (asc.) target positions: The computer and the user share the autonomy over the end position of path $\boldsymbol{f}_{p}$, according to the definition of $G_{1}$. The arbitration is done automatically by the computer according to the posterior probability (similarly to [17]) explained in section VI. This causes $\left.\boldsymbol{f}_{p}\right|_{s=1}$ to be attracted to intent-inferred targets, as illustrated in Fig. 2.

2. $G_{2}-$ Automatic motion (desc.) versus RVGF (asc.): The computer and the user share the autonomy over the position $\boldsymbol{p}(\boldsymbol{q})$ of the robot end effector, and its arbitration is manually performed according to $G_{2}$. Thus, the user can activate the automatic reactive approach motion BB by events triggered through the event HMI. The force SIM $\left(\hat{w}_{8_{2}}\right)$ was excluded from $G_{2}$ so that there is always reaction to forces. In addition, a quasi-trapezoidal velocity profile of $\dot{s}$ is achieved $\left(\hat{w}_{13_{2}}\right)$. Linear (with respect to time) rise and fall sections of this velociy profile are obtained by triggering an event that switches the value of $G_{2}$. Also, by defining $V_{p}(s)$ as a piecewise function that is constant at $s \leq s_{\text {dwell }}$ and linear from $s_{\text {dwell }}<s \leq 1$, the velocity profile autonomously decreases linearly (with respect to $s$ ) towards zero when reaching the object (see Fig. 8).

3. $G_{3}-R V G F$ (desc.) versus full user control (asc.): Arbitration over $\boldsymbol{p}(\boldsymbol{q})$ is done based on the distance $G_{3}=$ $\left\|\boldsymbol{p}(\boldsymbol{q})-\boldsymbol{f}_{p}(s)\right\|$. Thus, the user can escape from or come 
back to the RVGF by simply forcing the end effector away or driving it close to the RVGF.

4. $G_{4}$ - Idle (desc.) versus RVGF (asc.): The user has partial autonomy over $\boldsymbol{p}(\boldsymbol{q})$ by means of SIMs that incorporate teleoperation or kinesthetic HMIs. However, by choosing $G_{4}=\left\|\boldsymbol{v}_{\mathrm{hmi}}\right\|+\left\|k_{\text {gain }} \boldsymbol{C}_{a} \boldsymbol{F}_{\text {meas }}\right\|$, the impedance-like behavior related to the RVGF is deactivated automatically (i.e. nonlinear impedance) when the user is not interacting with the robot. This avoids drift when the user is not interacting with the robot (even if $e_{11} \geq r_{\text {tube }}(s)$ ).

5. $G_{5}$ - Teleoperation (desc.) versus force interaction (asc.): The end effector position $\boldsymbol{p}(\boldsymbol{q})$ can be driven manually by a teleoperation HMI. Nevertheless, when forces in the end effector are sensed, the corresponding autonomy can be automatically overtaken by the force SIM (to deal with humanrobot physical interaction or unexpected collisions).

TABLE II. Specification of five levels of autonomy and the involved weight factors. The descending and ascending weight factors $\hat{w}_{i_{k}}$ are displayed in separate columns. Subscript $i$ indicates the corresponding constraint (see Table I) and subscript $k$ indicates the corresponding arbitration parameter For all weights $\hat{w}_{i}$ whose factors $\hat{w}_{i_{k}}$ are not mentioned here: $a_{i}=0$ and $b_{i}>0$. For all the factors mentioned here: $\hat{w}_{i_{k}}^{\max }=1, \hat{w}_{i_{k}}^{\min }=0$ and $b_{i}=0$, unless stated otherwise.

\begin{tabular}{|c|c|c|}
\hline $\mathbf{G}_{\mathbf{k}}$ & Desc. Asc. & Description of the arbitration parameter \\
\hline$G_{1}$ & $\left\{\hat{w}_{4_{1}}\right\} \quad\left\{\hat{w}_{9_{1}}\right\}$ & $\begin{array}{l}\text { Manual vs automatic target positions } \\
\text { Generic-policy: } G_{1}=P\left(O_{\text {pred }} \mid \boldsymbol{x}_{\mathrm{hmi}}\right), G_{1}^{0}=P_{\min } \\
\text { and } G_{1}^{1}=P_{\max } \text {. }\end{array}$ \\
\hline$G_{2}$ & $\begin{array}{ll}\left\{\hat{w}_{13_{2}},\right. & \left\{\hat{w}_{7_{2}},\right. \\
\left.\hat{w}_{14_{2}}\right\} & \hat{w}_{11_{2}} \\
& \left.\hat{w}_{12_{2}}\right\}\end{array}$ & $\begin{array}{l}\text { Automatic motion vs RVGF } \\
\text { Time-policy: } b_{11}>0 \text { is a small value in order to } \\
\text { keep adapting } s \text {. }\end{array}$ \\
\hline$G_{3}$ & $\begin{array}{l}\left\{\hat{w}_{11_{3}},\{\}\right. \\
\left.\hat{w}_{12_{3}}\right\}\end{array}$ & $\begin{array}{l}\text { RVGF vs full user control } \\
\text { Generic-policy: } G_{3}=\left\|\boldsymbol{p}(\boldsymbol{q})-\boldsymbol{f}_{p}(s)\right\| \text {, where } G_{3}^{0} \\
\text { and } G_{3}^{1} \text { are threshold distances. }\end{array}$ \\
\hline$G_{4}$ & $\begin{array}{l}\left\{\hat{w}_{11_{4}},\right. \\
\left.\hat{w}_{12_{4}}\right\}\end{array}$ & $\begin{array}{l}\text { Idle vs RVGF } \\
\text { Generic-policy: } G_{4}=\left\|\boldsymbol{v}_{\mathrm{hmi}}\right\|+\left\|k_{\text {gain }} \boldsymbol{C}_{a} \boldsymbol{F}_{\text {meas }}\right\| \text {, } \\
\text { where } G_{4}^{0}=G_{4}^{1} \text { is a small velocity threshold. }\end{array}$ \\
\hline$G_{5}$ & $\left\{\hat{w}_{7_{5}}\right\} \quad\left\{\hat{w}_{85}\right\}$ & $\begin{array}{l}\text { Teleoperation vs force interaction } \\
\text { Generic-policy: } G_{5}=\left\|\boldsymbol{F}_{\text {meas }}\right\| \text {, where } G_{5}^{0} \text { and } G_{5}^{1} \\
\text { are small force thresholds. }\end{array}$ \\
\hline
\end{tabular}

\section{INTENT ESTIMATION}

In this section, we propose a simple Bayesian algorithm that estimates the intention of the user online. In the context of this work, which is assisting the user to reach the target object, the intention of the user is determined by selecting the most likely object $O_{\text {pred }}$ that the user intends to grasp with its corresponding probability $P\left(O_{\text {pred }} \mid \boldsymbol{x}_{\mathrm{hmi}}\right)$. The target position input $\boldsymbol{x}_{\mathrm{hmi}}$ is given explicitly (e.g. with a touchscreen) or implicitly (e.g. with gaze estimation) by the user through a target HMI. This approach is able to guide the user from the beginning of the execution, in contrast to approaches that estimate the intention based on user inputs coming from teleoperation HMIs.

We model the posterior probability that object $O_{k} \in \boldsymbol{O}$ is intended, given the input $\boldsymbol{x}_{\mathrm{hmi}}$, using Bayes' rule:

$$
P\left(O_{k} \mid \boldsymbol{x}_{\mathrm{hmi}}\right)=\frac{P\left(\boldsymbol{x}_{\mathrm{hmi}} \mid O_{k}\right) P\left(O_{k}\right)}{P_{r}+\sum_{O_{\mathrm{j}} \in \boldsymbol{O}} P\left(\boldsymbol{x}_{\mathrm{hmi}} \mid O_{\mathrm{j}}\right) P\left(O_{\mathrm{j}}\right)},
$$

where the joint probability $P_{r}=P\left(\boldsymbol{x}_{\mathrm{hmi}} \mid O_{\text {none }}\right) P\left(O_{\text {none }}\right)$ estimates when the user does not want to grasp any object (class $\left.O_{\text {none }}\right)$. The prior probabilities $P\left(O_{\mathrm{j}}\right)$ indicate known preferences of the user, or can all be assumed to be equal as done in section VII.

The conditional probabilities $P\left(\boldsymbol{x}_{\mathrm{hmi}} \mid O_{k}\right) \sim \mathcal{N}\left(\boldsymbol{x}_{k}, \boldsymbol{\Sigma}\right)$ are modelled as multivariate Gaussian distributions with covariance matrix $\boldsymbol{\Sigma} \in \mathbb{R}^{N_{x} \times N_{x}}$ and mean $\boldsymbol{x}_{k} \in \mathbb{R}^{N_{x}}$, where $N_{x}=2$ if the objects are assumed to be in a plane or $N_{x}=3$ otherwise. The position $\boldsymbol{x}_{k}$ of object $O_{k}$ is measured by a vision system. The covariance matrix $\boldsymbol{\Sigma}$ can be estimated from a dataset or, as we did in this work, by setting it to $\boldsymbol{\Sigma}=\sigma \boldsymbol{I}$ and heuristically selecting a convenient $\sigma$.

Let $d_{m}\left(\boldsymbol{x}_{\mathrm{hmi}}\right)=\sqrt{\left(\boldsymbol{x}_{\mathrm{hmi}}-\boldsymbol{x}_{\text {pred }}\right)^{\top} \Sigma^{-1}\left(\boldsymbol{x}_{\mathrm{hmi}}-\boldsymbol{x}_{\mathrm{pred}}\right)}$ denote the Mahalanobis distance, where $\boldsymbol{x}_{\text {pred }}$ is the position of the object corresponding to the maximum $P\left(\boldsymbol{x}_{\mathrm{hmi}} \mid O_{k}\right)$. Then, we define $P\left(\boldsymbol{x}_{\mathrm{hmi}} \mid O_{\text {none }}\right)$ as follows:

$$
P\left(\boldsymbol{x}_{\mathrm{hmi}} \mid O_{\text {none }}\right)=\frac{d_{\mathrm{sat}}-d_{\mathrm{min}}}{d_{\max }-d_{\min }},
$$

which has two effects: $(i)$ it saturates $d_{m}\left(\boldsymbol{x}_{\mathrm{hmi}}\right)$ through $d_{\mathrm{sat}}=\max \left(d_{\min }, \min \left(d_{\max }, d_{m}\left(\boldsymbol{x}_{\mathrm{hmi}}\right)\right)\right)$ with $d_{\min }$ and $d_{\max }$ parameters; and (ii) it performs a normalization such that $0 \leq P\left(\boldsymbol{x}_{\mathrm{hmi}} \mid O_{\text {none }}\right) \leq 1$. Because $d_{m}^{2}$ follows a $\chi^{2}$-distribution with $N_{x}$ DoFs, a confidence interval $\left[P_{\min }, P_{\max }\right]$ that determines $d_{\min }$ and $d_{\max }$ can be selected instead.

As a last step, we select the most likely object and its corresponding position by maximizing the posterior probability of this choice. Fig. 6 shows a bidimensional example with three arbitrary objects, two of which are sufficiently close to affect the autonomy regions of each another.

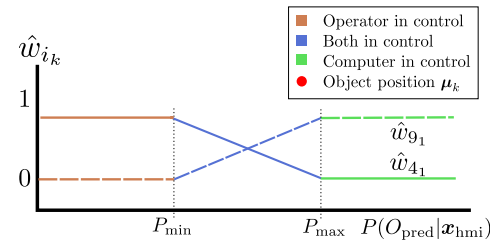

(a)

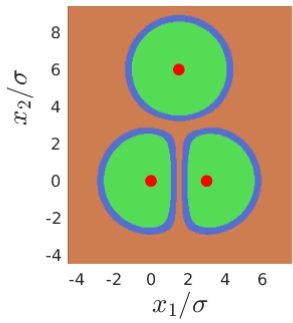

(b)
Fig. 6. Example of region depicting the levels of autonomy according to $G_{1}$, with $P_{\min }=0.6, P_{\max }=0.8$ and $P\left(O_{\text {none }}\right)=0.5$. (a) Weight factors vs posterior probability, (b) Regions for three objects with $N_{x}=2$ in dimensionless position coordinates $\boldsymbol{x}_{\mathrm{hmi}} / \sigma$, where $\boldsymbol{x}_{\mathrm{hmi}}=\left[\begin{array}{ll}x_{1} & x_{2}\end{array}\right]^{\top}$.

\section{VAlidation USE CASES}

Because of the focus on adaptability and reconfigurability of our framework, we chose to demonstrate three widely differing use cases rather than focussing on a user study to show the suitability of a particular configuration to a user group with specific mobility limitations. The configurations shown in Table III were designed to demonstrate the customization capabilities of our framework to comply with various mobility limitations and scenarios. They were not designed to be the optimal for specific mobility limitations.

In all cases, an able-bodied user was chosen from the authors. An off-the-shelf vision system was used for determining positions $\boldsymbol{x}_{k}$ of taught objects $O_{k}$, and these were used as inputs for the intent-inference algorithm. Also, the force $\boldsymbol{F}_{\text {meas }}$ 
TABLE III. Validation Use Cases

\begin{tabular}{|c|c|c|c|}
\hline & Use Case 1 & Use Case 2 & Use Case 3 \\
\hline Target User & Able-bodied & Paraplegic & Tetraplegic \\
\hline Robotic Platform & Franka Panda & Franka Panda & Kinova Gen 3 \\
\hline Teleop. HMI & None & SpaceMouse & None \\
\hline Kinesthetic HMI & Torque sensors & None & None \\
\hline Target HMI & $\begin{array}{l}\text { 3D Camera: hand } \\
\text { pointing }\end{array}$ & SpaceMouse & Eye tracker \\
\hline Events HMI & Voice commands & Buttons & Blink detector \\
\hline Tube Shape Source & $\begin{array}{l}\text { Human } \\
\text { demonstrations }\end{array}$ & Planned paths & Modeled paths \\
\hline
\end{tabular}

(a)

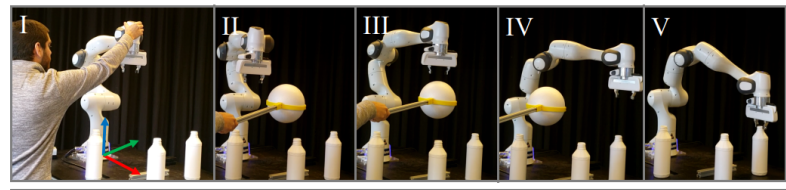

(b)



Fig. 7. Snapshots of the use cases, whose target user and configuration is defined in Table III. (a) Use case 1, (b) use case 2 and (c) use case 3.

at the robot end effector was estimated by means of measured joint torques.

Fig. 7a shows snapshots of use case 1 and Fig. 8 shows relevant control signals that describe the obtained behavior. First, the user indicated the desired target with the fingertip, which was detected through the vision system. Then, from time instances $I$ to $I I$, the user pulled the position of the end effector $\boldsymbol{p}(\boldsymbol{q})$ away from the RVGF defined by path $\boldsymbol{f}_{\mathrm{p}}$ in (5) by means of the kinesthetic HMI (RVGF versus full user control). At $I I$, automatic motion is activated through a voice command after an obstacle is brought into the scene. From II the progress variable $s$ advances with a quasi-trapezoidal velocity profile, making the orientation angle $f_{\hat{\theta}}(s)$ (see subsection IV-D) also advance. Meanwhile, $\boldsymbol{p}(\boldsymbol{q})$ slowly converges to $\boldsymbol{f}_{\mathrm{p}}$ following first order dynamics. At III, the minimum distance $d_{i}$ from the vision-detected obstacle to the path reaches its threshold $d_{\text {th }}$ (collision avoidance SIM). This causes path $\boldsymbol{f}_{\mathrm{p}}$ to adapt its shape to circumvent the obstacle. Then, at $I V$, the obstacle is retracted and $f_{\mathrm{p}}$ returns elastically to match $\boldsymbol{f}_{\mathrm{lm}}$ (constraint 3 ) and grasps the intent-inferred object at $V$.

Fig. 7b shows snapshots of use case 2 and Fig. 9 shows relevant control signals that describe the obtained behavior. A SpaceMouse was used to teleoperate the robot and to implicitly select the target object. The computer's autonomy over $\left.\boldsymbol{f}_{p}\right|_{s=1}$ (indicated by $\hat{w}_{9_{1}}$ ) is continuously shifted between $I$ to $I I$, due to $\boldsymbol{x}_{\mathrm{hmi}}$ being driven by the user. The change of $\boldsymbol{x}_{\mathrm{hmi}}$ caused the shape of the RVGF to be adapted towards new targets by changing the latent variables $\chi_{\mathrm{lv}}$ defined in (4). From II to $I I I$, the robot was teleoperated by the user who is assisted by the RVGF. At III, the user shifted $\boldsymbol{x}_{\mathrm{hmi}}$ which dragged away the RVGF from $\boldsymbol{p}(\boldsymbol{q})$, causing a shift towards full user control

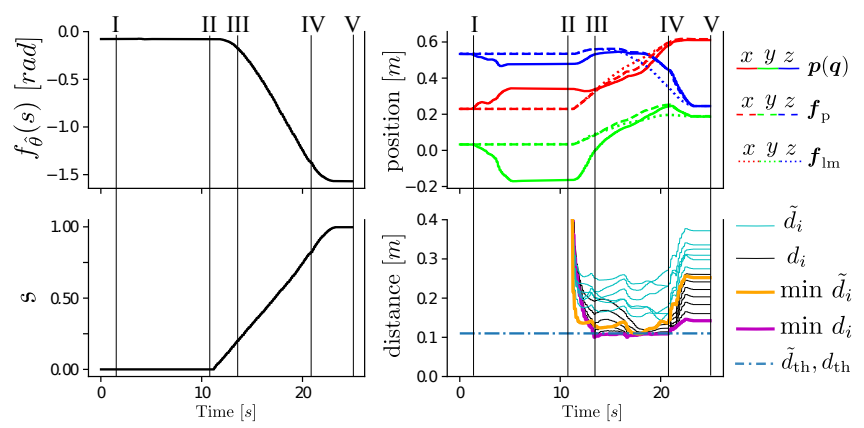

Fig. 8. Control signals of an experiment performed for validation use case 1 , where the time instances denoted with roman numbers correspond to the snapshots in Fig. 7a.
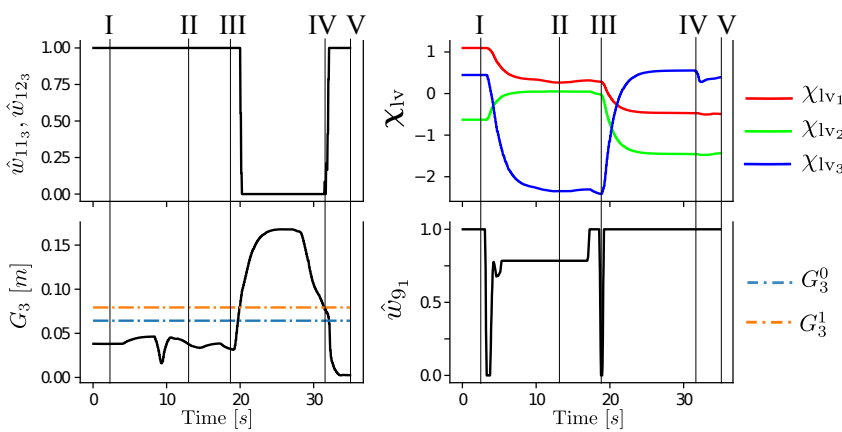

Fig. 9. Control signals of an experiment performed for validation use case 2 , where the time instances denoted with roman numbers correspond to the snapshots in Fig. 7b.

by means of the weight factors $\hat{w}_{11_{3}}$ and $\hat{w}_{12_{3}}$ (see $G_{3}$ signal explained in Table II). The user freely teleoperated the robot until $I V$, where $G_{3}$ decreased to a low value, re-enabling the RVGF towards the grasp of the object at $V$.

Fig. $7 \mathrm{c}$ shows snapshots of use case 3 , where a user is able to command the robot with a head-mounted eye-tracker [18]. In $I$, the user drives $\boldsymbol{x}_{\mathrm{hmi}}$ with the gaze (green circle), which is only updated when fixating on an area delimited by QR tags. The location and dimensions of that area were determined by the vision system, and the eye-tracker provided the gaze expressed in a local frame attached to the QR tags, allowing relocation of the user. After the user confirmed that the target was updated correctly through a screen, at $I I$ he activated the automatic motion with two consecutive forced blinks. At III, the robot reached the target location with an added offset, so that the user could correct if necessary. Finally, at $I V$ the user commanded the grasp by a long blink gesture.

\section{DISCUSSION AND CONCLUSION}

This work provides a framework consisting of tools for defining adaptable reactive behaviors that allocate user or computer autonomy over robot-related or task-related variables. The modulation of the autonomy (i.e. arbitration) over each variable can be done systematically via two types of weight shifting, which enable online adaptation of the levels of autonomy. These modulation can be performed in function of relevant computer-controlled or user-controlled variables in a flexible and composable way.

During the validation use cases, the modularity and composability of the building blocks enabled the easy reconfiguration 
of the system to comply to the mobility limitations of ablebodied, paraplegic, and tetraplegic targeted users. This was achieved by separating (i) the sensor-interaction, specified using specific SIMs suited to different types of HMIs, from (ii) the specification of nominal actions and the deviation from these using the RAMs. The composition and modulation of these two types of building blocks results in the described levels of autonomy. This stands in contrast to the state of the art, where the resulting behavior that combines task and sensor-information is directly implemented in e.g. impedancelike behaviors, which makes it more difficult to reconfigure to new types of user input.

The used constraint-based approach also bears similarities with the autonomous dynamical systems described in [19] for human-robot interactive applications. For instance, in the case of the automatic reactive approach motion $\mathrm{BB}$, the only explicit time dependency is contained within the weights, which are used to give the user the possibility of activating/deactivating its resulting behavior at arbitrary time instances. Once the activation has been made, the weights remain constant and the dynamical system becomes autonomous (i.e. time-invariant). This, in combination with other building blocks such as the automatic orientation path, allows us to easily specify autonomous dynamical systems governing reactive behaviors in $S E(3)$, which are otherwise cumbersome to establish and to adapt to other applications.

The proposed intent-inference algorithm enables the use of target HMIs with high uncertainty such as an eye-tracker. Its online inference capability leverages the reactiveness of our framework by allowing adaptation to changes of intention. The class $O_{\text {none }}$ is explicitly included such that the user can automatically be granted with full autonomy over $\left.\boldsymbol{f}_{p}\right|_{s=1}$ when, for example, the vision system fails to detect an object that the user wants to reach. This would allow the user to still reach the goal at the expense of more effort.

The proposed modular specification of assistive tasks and arbitration of autonomy facilitates the potential combination with other intent estimation approaches based on teleoperation HMI inputs, such as the ones proposed in [17], [20] and [21].

The reactiveness of our framework can also enable a safe teleoperation of the robot within an unstructured environment, adapting compliantly to dynamic obstacles and external forces. We are convinced that the offered reconfigurability could also be used for other tasks such as pouring, insertion, or completely different scenarios, such as teleoperation in hazardous environments or human-robot collaborative industrial applications. Future assistive devices could benefit from this work and impact the quality of life of people that require robotic assistance. Of course, this work needs to be followed up with a user study to evaluate the response and acceptance of subjects with limitations of their mobility. The offered reconfigurability and adaptability enables user-specific customization that is essential to obtain the acceptance of a larger user community.

\section{REFERENCES}

[1] D. Kim, R. Hazlett-Knudsen, H. Culver-Godfrey, G. Rucks, T. Cunningham, D. Portee, J. Bricout, Z. Wang, and A. Behal, "How autonomy impacts performance and satisfaction: Results from a study with spinal cord injured subjects using an assistive robot," IEEE Transactions on
Systems, Man, and Cybernetics - Part A: Systems and Humans, vol. 42, no. 1, pp. 2-14, 2012.

[2] M. Wang, A. A. Kogkas, A. Darzi, and G. P. Mylonas, "Free-view, 3d gaze-guided, assistive robotic system for activities of daily living," in 2018 IEEE/RSJ International Conference on Intelligent Robots and Systems (IROS), 2018, pp. 2355-2361.

[3] D. P. Losey, K. Srinivasan, A. Mandlekar, A. Garg, and D. Sadigh, "Controlling assistive robots with learned latent actions," in 2020 IEEE International Conference on Robotics and Automation (ICRA), 2020, pp. 378-384.

[4] F. Dimeas, F. Fotiadis, D. Papageorgiou, A. Sidiropoulos, and Z. Doulgeri, "Towards progressive automation of repetitive tasks through physical human-robot interaction," in Human Friendly Robotics, F. Ficuciello, F. Ruggiero, and A. Finzi, Eds. Cham: Springer International Publishing, 2019, pp. 151-163.

[5] D. Aarno, S. Ekvall, and D. Kragic, "Adaptive virtual fixtures for machine-assisted teleoperation tasks," in Proceedings of the 2005 IEEE International Conference on Robotics and Automation, 2005, pp. 11391144.

[6] M. Selvaggio, G. A. Fontanelli, F. Ficuciello, L. Villani, and B. Siciliano, "Passive virtual fixtures adaptation in minimally invasive robotic surgery," IEEE Robotics and Automation Letters, vol. 3, no. 4, pp. 3129 3136,2018

[7] M. Ewerton, O. Arenz, and J. Peters, "Assisted teleoperation in changing environments with a mixture of virtual guides," Advanced Robotics, vol. 34 , no. 18 , pp. 1157-1170, 2020.

[8] T. Stoyanov, R. Krug, A. Kiselev, D. Sun, and A. Loutfi, "Assisted telemanipulation: A stack-of-tasks approach to remote manipulator control," in 2018 IEEE/RSJ International Conference on Intelligent Robots and Systems (IROS), 2018, pp. 1-9.

[9] G. Quere, A. Hagengruber, M. Iskandar, S. Bustamante, D. Leidner, F. Stulp, and J. Vogel, "Shared control templates for assistive robotics," in 2020 IEEE International Conference on Robotics and Automation (ICRA), 2020, pp. 1956-1962.

[10] A. Dragan, K. Lee, and S. Srinivasa, "Teleoperation with intelligent and customizable interfaces," Journal of Human-Robot Interaction, vol. 2, no. 2, pp. 33 - 57, June 2013.

[11] D. Gopinath, S. Jain, and B. D. Argall, "Human-in-the-loop optimization of shared autonomy in assistive robotics," IEEE Robotics and Automation Letters, vol. 2, no. 1, pp. 247-254, 2017.

[12] E. Aertbeliën and J. De Schutter, "ETaSL/eTC: A constraint-based task specification language and robot controller using expression graphs," in IEEE Int. Conf. on Intel. Robots and Systems, 2014, pp. 1540-1546.

[13] C. A. Vergara, J. De Schutter, and E. Aertbeliën, "Combining imitation learning with constraint-based task specification and control," IEEE Robot. and Autom. Lett., vol. 4, no. 2, pp. 1892-1899, April 2019.

[14] C. A. Vergara, S. Iregui, J. De Schutter, and E. Aertbeliën, "Generating reactive approach motions towards allowable manifolds using generalized trajectories from demonstrations," in IEEE Int. Conf. on Intel. Robots and Systems, 2020, pp. 9697-9704.

[15] O. Brock and O. Khatib, "Elastic strips: A framework for motion generation in human environments," The International Journal of Robotics Research, vol. 21, no. 12, pp. 1031-1052, 2002.

[16] D. P. Losey, C. G. McDonald, E. Battaglia, and M. K. O'Malley, "A Review of Intent Detection, Arbitration, and Communication Aspects of Shared Control for Physical HumanRobot Interaction," Applied Mechanics Reviews, vol. 70, no. 1, 02 2018, 010804.

[17] A. D. Dragan and S. S. Srinivasa, "A policy-blending formalism for shared control," The International Journal of Robotics Research, vol. 32, no. 7, pp. 790-805, 2013.

[18] M. Kassner, W. Patera, and A. Bulling, "Pupil: An open source platform for pervasive eye tracking and mobile gaze-based interaction," in Adjunct Proceedings of the 2014 ACM International Joint Conference on Pervasive and Ubiquitous Computing, ser. UbiComp '14 Adjunct. New York, NY, USA: ACM, 2014, pp. 1151-1160.

[19] M. Khoramshahi and A. Billard, "A dynamical system approach for detection and reaction to human guidance in physical human-robot interaction," Autonomous Robots, vol. 44, no. 8, pp. 1411-1429, Nov 2020.

[20] S. Jain and B. Argall, "Probabilistic human intent recognition for shared autonomy in assistive robotics," J. Hum.-Robot Interact., vol. 9, no. 1, Dec. 2019.

[21] K. Muelling, A. Venkatraman, J.-S. Valois, J. E. Downey, J. Weiss, S. Javdani, M. Hebert, A. B. Schwartz, J. L. Collinger, and J. A. Bagnell, "Autonomy infused teleoperation with application to brain computer interface controlled manipulation," Autonomous Robots, vol. 41, no. 6, pp. 1401-1422, Aug 2017. 